\title{
Real-Time Congestion Management in Distribution Networks by Flexible Demand Swap
}

\author{
Huang, Shaojun; Wu, Qiuwei
}

Published in:

IEEE Transactions on Smart Grid

Link to article, DOI:

10.1109/TSG.2017.2655085

Publication date:

2017

Document Version

Peer reviewed version

Link back to DTU Orbit

Citation $(A P A)$ :

Huang, S., \& Wu, Q. (2017). Real-Time Congestion Management in Distribution Networks by Flexible Demand Swap. IEEE Transactions on Smart Grid, 9(5), 4346 - 4355. https://doi.org/10.1109/TSG.2017.2655085

\section{General rights}

Copyright and moral rights for the publications made accessible in the public portal are retained by the authors and/or other copyright owners and it is a condition of accessing publications that users recognise and abide by the legal requirements associated with these rights.

- Users may download and print one copy of any publication from the public portal for the purpose of private study or research.

- You may not further distribute the material or use it for any profit-making activity or commercial gain

- You may freely distribute the URL identifying the publication in the public portal

If you believe that this document breaches copyright please contact us providing details, and we will remove access to the work immediately and investigate your claim. 


\title{
Real-Time Congestion Management in Distribu- tion Networks by Flexible Demand Swap
}

\author{
Shaojun Huang, Student Member, IEEE, Qiuwei Wu, Senior Member, IEEE
}

\begin{abstract}
In addition to the day-ahead congestion management in distribution networks, the real-time congestion management is very important because many unforeseen events can occur at the real operation time, e.g. loss of generation of distributed energy resources (DERs) or inaccurate forecast of energy consumption or production. Flexibility service from demand will be a good option to solve the real-time congestions if the cost of activating the flexibility service is fully addressed. This paper proposes a new method, namely "swap", to employ the flexibility service from electric vehicles (EVs) and heat pumps (HPs) for real time congestion management. The swap method can maintain the power balance of the system and avoid the imbalance cost of activating the flexibility service. An algorithm for forming swaps through optimal power flow (OPF) and mixed integer linear programming (MILP) is proposed to implement the swap method. Case studies were carried out to validate the efficacy of the proposed swap method for real time congestion management and the proposed algorithm for forming swaps. The settlement process for the swaps in different markets is analyzed.
\end{abstract}

Index Terms-- Congestion management, distributed energy resources (DERs), distribution system operator (DSO), electric vehicles (EVs), flexibility service, heat pumps (HPs).

\section{NOMENCLATURE}

\section{Parameters}

$\mathcal{N}$

$\mathcal{N}_{1}$

$\mathcal{N}_{2}$

$\mathcal{I}$

$\mathcal{I}^{*}$

$\mathcal{T}$

$\mathcal{T}^{*}$

$D$

$R$

$S_{1}$

$S_{2}$

$X$

$\underline{V}$

$V_{0}$

$Y_{L L}$ set of the nodes in the distribution network set of the load points in $S_{1}$ set of the load points in $S_{2}$ set of allowed swaps set of selected swaps from $\mathcal{I}$ set of overall planning periods set of selected periods from $\mathcal{T}$ for forming swaps power transfer distribution factor (PTDF) real element of matrix $Z$ one side of the swap the second side of the swap, to balance $S_{1}$ imagine element of matrix $Z$ voltage lower limit voltage at node 0 , the secondary side of the high voltage transformer the matrix obtained by removing the first row and

The work was supported by the EU Seventh Framework Programme (FP7) through the 'Ideal Grid for All (IDE4L)' project.

S. Huang, and Q. Wu are with the Center for Electric Power and Energy (CEE), Department of Electrical Engineering, Technical University of Denmark (DTU), 2800 Kgs. Lyngby, Denmark (e-mail: shuang@elektro.dtu.dk; qw@elektro.dtu.dk).

column of the nodal admittance matrix

\section{Z}

$f$

$\tilde{f}$

$p$

$p_{j^{\prime} t}^{b}$

$q_{j^{\prime} t}^{b}$

$r_{1}$

$r_{2}$

$S$

$t_{1}$

$t_{1}$

$\delta$

Variables

$X$

$y$

Abbr.

ADMM alternating direction method of multipliers

BRP balance responsible party

DR demand response

DT dynamic tariff

DSO distribution system operator

DRX demand response exchange

EV electric vehicle

FCH flexibility clearing house

HP heat pump

ISO independent system operator

LMP locational marginal price

MILP mixed integer linear program

OPF optimal power flow

$\mathrm{RP} \quad$ regulation price

SOC state of charge

TSO transmission system operator

\section{INTRODUCTION}

TN deregulated power systems, electricity prices play an 1 important role to balance the production of renewable energy, such as wind power and solar power, and the consumption of flexible demands, such as electric vehicles (EVs) and heat pumps (HPs) equipped with energy storages. Both the renew- 
able energy and the flexible demands have been largely introduced into the power system. This trend will continue to achieve independence from fossil fuels and reduce $\mathrm{CO}_{2}$ emissions. Congestion management is one of the challenges that the system operators will face with high penetration of renewable energy and flexible demands.

Congestion may occur in transmission grids and result in different locational prices in the locational marginal pricing (LMP) systems or different zonal prices in the zonal pricing systems. Congestion may also occur in distribution networks, especially when more and more distributed energy resources (DERs) and flexible demands are integrated at the distribution level.

\section{A. Review of Congestion Management Methods by DR}

Demand response (DR) is very promising in dealing with the congestion management. DR has two types, namely the price-based DR and the incentive-based DR. In recent research of congestion management at the distribution level, which is the focus of this paper, several methods based on the day-ahead market have been proposed. The distribution LMP (DLMP) [1]-[3] can be employed to trigger the dispatchable distributed generators to alleviate the congestion in heavy loaded feeders, while the dynamic tariff (DT) method [4]-[7] and the dynamic subsidy method [8] can motivate aggregators to shift the flexible demands from peak hours at specific nodes. Based on the dual decomposition and alternating direction method of multipliers (ADMM) technique, the grid price methods [9]-[13] work similarly to the DT method but require iteration processes between the distribution system operator (DSO) and aggregators. All the aforementioned methods are prices-based DR programs which can be used by the DSO to solve congestions. They have a common feature that the final prices at congestion hours are higher than those without congestions; therefore, the flexible demands, who want to minimize the costs, will shift to off-peak hours and relieve the congestions.

For the incentive-based DR, the authors of [14] has proposed a coupon incentive-based congestion management method, and in [15], an optimal coordination and scheduling method via monetary incentives is proposed. Both methods require iterative processes to determine the final incentives and demand changes. Flexibility clearing house (FCH) [16]-[18] and a more advanced version — DR exchange (DRX) platform [19]-[21], aim at establish a pool-based market just for DR. All these incentive-based DR programs have a common feature that they need to clearly quantify the changes of the flexible demand and then reward the changes accordingly.

As important as the congestion management in the day-ahead market where majority of the energy production and consumption is planned, the congestion management close to the operation time ( 5 to 60 minutes ahead of the operation time) because of the forecast error or the component failure should be handled by the DSO as well. This congestion management is referred as real-time congestion management.

In [22], a real-time market (5 minutes ahead of the operation time) architecture for European electricity markets is proposed, where the flexible demands are programmed to be simple price-responsive loads. The authors of [23] has proposed a DR model for the real-time market. However, the real-time market operates independently from other markets, i.e. customers participating in the real-time market will not participate in the day-ahead market. Therefore, it will not be considered in this paper since we assume that the day-ahead market continues to be the major market in the foreseeable future. The complexity of employing DR for the real-time congestion management lies in the fact that the flexible demands have participated in the day-ahead market and made energy plans already. Therefore, it is not suitable to employ the price-based DR to motivate and change the flexible demands. On the other hand, the incentive-based DR is naturally suitable for real-time congestion management because the flexible demands are rewarded by their 'changes'. The changes are measured as the difference between the actual demands and those planed at the day-ahead market.

\section{B. Motivation}

This paper proposes a new incentive-based DR program for real-time congestion management and can overcome the drawbacks in the existing DR programs. There are two important aspects should be taken into account when designing a DR program for the real-time congestion management. The first one is the system balance, which is achieved after the clearing of the day-ahead market and should be maintained after employing any DR program henceforth because the system balance is critical for the system security. And when there are system balance issues, additional costs to the DR providers may occur due to the settlement of the system imbalance according to the regulation market. The second one is the rebound effect of the flexible demands, which can cause system balance issues and additional cost in future hours. The aggregators, as the provider of flexibility services, will not be willing to provide such services if the cost of them is not known or very difficult to forecast. The cost refers to the settlement of system imbalance and the energy cost due to the future batteries/temperature recovery needs.

Either or both of the two important aspects have not been studied in the existing incentive-based DR programs. For instance, the rebound effect is not considered in the coupon incentive-based DR program [14] because the intertemporal feature of the flexible demands isn't considered in its model. The rebound effect is considered in the DR model via monetary incentives [15], however, the system imbalance issue and the associated costs are not studied.

In both concepts, namely the FCH and DRX, it is not difficult to create a market for buying and selling DR; the difficulty lies in how to motivate the aggregators to participate and provide DR because it is difficult for them to estimate the cost due to the system balance settlement and the rebound effect. The rebound effect may also lead to congestions in a future time since the aggregators have no information of the grid conditions. In addition, the market clearing of the FCH and DRX depends very much on the submissions of the DR bids; the market may not be cleared or achieve an optimal solution because the DR bids are not 'smart' enough. For instance, the 
congestions may be located in one point of the distribution grid, but the DR bids are all located in other points of the grid. Too much burden is put on the aggregators/customers, which is not good for encouraging them to participate in the market. Therefore, this paper proposes a method from other perspectives and let the DSO take the initiative since it has both the grid information and the ability to forecast the congestions and flexible demands.

Taking into account the imbalance issue and the costs of providing flexibility services, this paper proposes a real-time congestion management method by swapping the consumption of the flexible demands, i.e. the EV charging/discharging and the HP consumption increase/decrease. In the proposed method, the 'swap' occurs both temporally and spatially. The spatial swap helps maintain the system balance and avoid the cost of the system imbalance settlement. For instance, a decrease of consumption at one node is compensated by an increase with the same amount at another node; as such, the system balance is not affected. The temporal swap can help avoid the rebound effect; because the flexible demands participating in the DR program will have the chance to restore their batteries or the temperature level of their households with the temporal swap. Taking either side of the swap and providing the DR, the aggregators can be rewarded by the DSO according to the amount of the provided flexibility.

The 'buy back' method [24] based on the regulation market for transmission line congestion management by the transmission system operator (TSO) is also a swap method, but it only exchange the consumption/generation spatially, not temporally. Since it is initiated by the TSO, there is no system balance issue. The players in the 'buy back' method are often large generators and they normally don't have the rebound effect. Therefore, the situation of the congestion management by the TSO and the DSO are quite different.

The benefits of the proposed swap method are summarized as: (1) No system balance issue; (2) No rebound effect; (3) No unforeseen costs due to the system imbalance and the rebound effect; (4) The reward/cost for the aggregators/DSO is clear. The main contribution of this paper is proposing the swap method for real-time congestion management and the algorithm for forming the swaps through optimal power flow (OPF) and mixed integer linear programming (MILP).

The paper is organized as follows. The Nordic electricity markets are described in Section II. The method of swapping the EV charging and HP consumption is presented in Section III. In Section IV, the algorithm for forming swaps is presented. In Section V, case studies are presented and discussed, followed by conclusions.

\section{DESCRIPTION OF EXISTING MARKET}

In this section, the relevant electricity markets are described in order to analyze the cost of providing flexibility services. The Nordic electricity market is introduced with details, which represents zonal-price market prevailing in Europe.

\section{A. Day-ahead Market}

The day-ahead market is the most important energy market in the Nordic electricity market, as the majority of the electricity is traded in this market. The market players submit their bids into a pool, and the market operator clears the market. After the clearance, the market players will receive the accepted bids, and the hourly zonal prices as well as the hourly system prices will be published. If there is no congestion between zones, the zonal prices will be equal to the system prices.

Due to the participation of renewable energy, the electricity price fluctuates and is largely dependent on the weather forecast. The aggregators of flexible demands should buy the electricity from this market to fulfil the daily consumption requirement of the flexible demands.

However, any deviation from the day-ahead energy plans, i.e. the difference between the actual production or consumption and the day-ahead schedule, will incur additional cost unless it is helping the system balance according to the results of the regulation power market. Providing flexibility services, if activated, will cause such deviations and therefore will be subject to potential additional cost, which should be taken care of.

\section{B. Intra-day Market}

The intra-day market is a bilateral contract market and is closed at one hour before the actual operation time. The bids not selected at the day-ahead market can be traded here. Market players can make contracts here and avoid the potential cost of causing system imbalance if they have failed components or inaccurate forecast and need balance help from other participants. Due to the limited capacity and lifetime of storage systems of flexible demands, the flexibility services are not suitable to be traded here.

\section{Regulating Power Market}

The regulating power market is an auction market managed by the transmission system operator (TSO) where the up regulation (increase production or reduce consumption with duration of e.g. 30 60 minutes) and the down regulation (decrease production or increase consumption) are separated. The market is closed at 45 minutes before the operation time and the bids cannot be modified (price and quantity) after the closure.

The bids are activated in a sequence according to their prices. For a given hour, the up regulation price (RP) is set as the last activated up regulation bid and the down RP is set as the last activated down regulation bid. It is possible that both up regulation bids and down regulation bids are activated; then a common RP is determined to be one of the up and down regulation price depending on the net effect of the total up and down regulation power. For instance, if the net effect of the total regulation power is up regulation, the common regulation price is determined to be the up regulation price. In this case, all the up regulation bids are settled at the common RP while all the down regulation bids are settled by pay-as-bid.

The imbalance of each balance responsible party (BRP), i.e. the difference between the metered data and the scheduled or notified plan resulting from spot market and regulating market, will be settled after the operation time. The imbalance that contributes to the system total imbalance will be settled at the common RP, and the imbalance that is in the opposite direction 
of the system total imbalance will be settled at the day-ahead spot price. This settlement method is referred as two-price settlement. If one-price settlement applies, the imbalance is always settled at the common RP.

Aggregators of flexible demands can participate in the regulating power market, however, it is suggested that the bids should be checked by the DSO to ensure the security of the distribution networks before submitted to the regulating power market.

\section{Ancillary Service Market}

Ancillary services, including primary reserve, secondary reserve, manual reserve, and black-start capacity, are traded in this market. EVs are believed to be a good source for providing ancillary services due to the ability of quick response. Bids from the aggregators should be first checked by the DSO to ensure the security of the distribution networks.

\section{E. Other Types of Market}

In section II.A-D, the zonal price market has been described with details. Employing swap for real-time congestion management is not only suitable for zonal price markets, but also suitable for single price markets, e.g. regulated electricity market in China, and the nodal price markets, e.g. many markets in North America. The technical issues of using swaps, e.g. the limitation of distribution networks and transmission networks, are the same for the three types of markets. The economic issues, i.e. the settlements of the swaps, will depend on the types of the markets. This will be further illustrated in case studies in subsection V.B. More detailed discussion of employing the swap method in single price markets or the nodal price markets will be carried out in our future work.

\section{METHOD OF SWAPPING}

Flexibility services, e.g. charging/discharging batteries of EVs and increasing/decreasing consumption of HPs, can be employed to resolve congestions in distribution networks. However, it must be done with the coordination of the relevant electricity markets, such as day-ahead spot market, regulating power market, and ancillary services market, such that the settlement rules of these markets are considered and the power system security/balance is ensured. In this section, the proposed method of swapping EV or HP consumption is described, which can help the aggregators provide flexibility services without causing system imbalance and at the same time avoid additional cost in the settlement of other associated markets.

\section{A. Swap within One DSO}

If the congestion is not at the connection point of the distribution network to the transmission grid, it is possible that the congestion can be resolved by a swap within the same DSO.

One side of a swap $\left(S_{1}\right)$ offered by the DSO can be defined as,

$$
S_{1}:\left\{L P_{j} \mid j \in \mathcal{N}_{1}\right\}, t_{1},-p, t_{2},+p,
$$

where set $\left\{L P_{j} \mid j \in \mathcal{N}_{1}\right\}$ represents the joint of load points where the flexibility service is needed, $t_{1}$ is the time period (when and how long) that the flexibility is needed, $-p$ means a consumption decrease with the total amount $p, t_{2}$ is the time period that the opposite flexibility is needed, $+p$ means a consumption increase with the total amount $p$, which is the opposite flexibility. The opposite flexibility is to give the aggregator a chance to restore the storage system associated with the flexibility, e.g. maintain the state of charge (SOC) level of the batteries of EVs.

The other side of the swap $\left(S_{2}\right)$ is defined as,

$$
S_{2}:\left\{L P_{j} \mid j \in \mathcal{N}_{2}\right\}, t_{1},+p, t_{2},-p,
$$

which means a consumption increase of the total amount $p$ is needed at $t_{1}$ and a consumption decrease of the total amount $p$ is needed at $t_{2}$ at any or a joint of the load points in $\left\{L P_{j} \mid j \in \mathcal{N}_{2}\right\}$. One aggregator can take one side of a swap and the other aggregator can take the other side. It is allowed that one aggregator takes both sides of a swap as long as it has the capacity of the required flexibility service.

Without losing generality, a distribution network (Fig. 1) from the Roy Billinton Test System (RBTS) [25] is employed to illustrate how the swap method can be used to handle real-time congestions in distribution networks. For instance, the DSO finds that there will be congestion, e.g. overloading by $p$, in 15 minutes at line L3 at Feeder 1, and the congestion will last for 30 minutes. The time information $\left(t_{1}\right)$ is written as "hour: minute, duration in minutes”, i.e. “18:00, 30”. By forecast, the DSO finds that there is enough free capacity of line L1 and the other feeders at $t_{1}$. The DSO also finds that there is enough free capacity at $t_{2}$ to perform the opposite flexibility. Then, it will raise an offer requesting a consumption decrease, written as,

$$
S_{1}:\left\{L P_{2-7}\right\}, t_{1},-p, t_{2},+p .
$$

At the same time, it will raise an opposite offer requesting a consumption increase, written as,

$$
S_{2}:\left\{L P_{1}, L P_{11-38}\right\}, t_{1},+p, t_{2},-p .
$$

The two offers form a swap. If both offers are taken by the aggregators, the congestion at L3 can be solved. It can be seen that the net change of consumption is zero at both $t_{1}$ and $t_{2}$, implying that the system balance is not influenced by the activation of the flexibility services.

The economics of the above swap is analyzed as follows. Firstly, the imbalance settlement described in section II.C is analyzed. Assume that the one-price settlement is applied and the regulation prices at $t_{1}$ and $t_{2}$ are $r_{1}$ and $r_{2}$, respectively. The first aggregator $\left(A_{1}\right)$, who takes $S_{1}$, should pay: $p \delta\left(r_{1}-r_{2}\right)$, while the second aggregator $\left(A_{2}\right)$, who takes $S_{2}$, should pay: $p \delta\left(-r_{1}+r_{2}\right)$, where $\delta$ is the duration of the swap. As a whole, the two aggregators do not pay anything to the independent system operator (ISO) or TSO due to the imbalance. But one aggregator needs to pay another. However, it is assumed that the aggregators have a special agreement with the DSO that no one would have profit or loss due to the settlement of imbalance resulting from the swap. Hence, with this special agreement, 
the aggregators pay zero in the imbalance settlement. The DSO will help to neutralize the profit or loss of the aggregators either by notifying the ISO such that the two aggregators need not to be involved in the imbalance settlement or by collecting profit from one aggregator and covering the loss of another.

Secondly, the settlement of the swap is analyzed. Assume that the price of the swap offered by the DSO is $s$. Both $A_{1}$ and

$A_{2}$ will receive the payment from DSO by the amount $p \delta s$, which is the profit from providing flexibility services. It should be noted that either side of the swap can be shared by several aggregators if one aggregator does not have enough flexibilities and the profit will be shared accordingly.

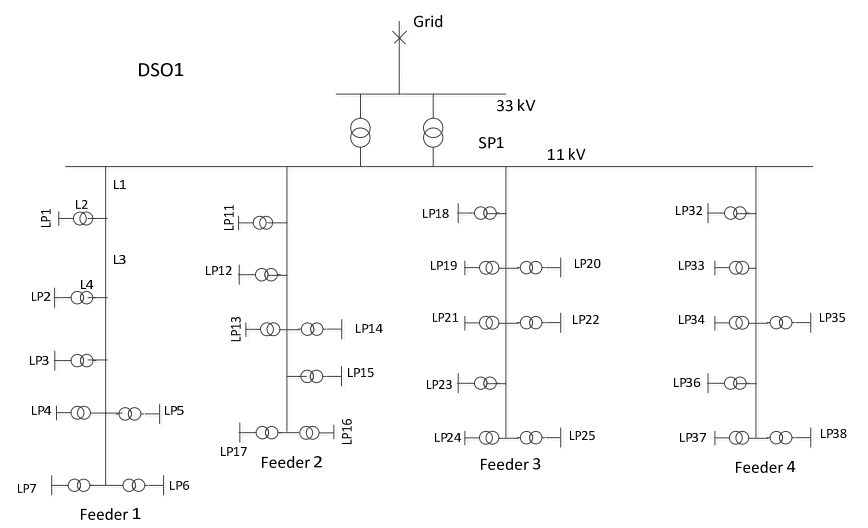

Fig. 1. Single line diagram of the distribution network

\section{B. System-wide Swap}

If there is not enough free capacity to implement $S_{2}$ inside the same distribution network which faces congestion issue identified by the DSO, denoted as $D_{1}$, it will need to seek help from other DSOs and the TSO. If a neighboring DSO is able to help $D_{1}$, the transmission limitation between different price zones does not need to be considered; otherwise, it needs to apply from relevant TSOs for the transmission capacity, which is free to use within the limitation, and seek the help from a remote DSO. In either case, if the requested free capacity to implement $S_{2}$ is identified in one or several other distribution networks, the system-wide swap can be formed, with $S_{1}$ implemented in the distribution network of $D_{1}$. The settlement of the system-wide swap is similar to the swap within one DSO since the help from the other DSOs and the TSO is "free".

\section{Procedure of Swap Market}

The concept of the swap market is shown in Fig. 2. "Swap" is a form of flexibility service proposed in this paper to solve real-time congestions without causing imbalance to the transmission network. The procedure is described below.

1) The DSO identifies the congestion problem within its network with at least 5 minutes in advance, such that there is enough time to set up a swap market.

2) The DSO identifies the free capacity to implement $S_{1}$ within its network.

3) The DSO identifies the free capacity to implement $S_{2}$ within its network; if not successful, it will ask help from a neighboring DSO; if not successful, it will ask help from a remote DSO within the allowed transmission capacity verified by the TSO.

4) If the free capacity to implement both $S_{1}$ and $S_{2}$ is found, the swap can be formed and published to the swap market by the DSO.

5) The aggregators can take the either side of the swap or both according to their flexibility service capacity.

6) The DSO confirms that both sides of the swap are taken by aggregators; otherwise, it will cancel the swap.

7) The aggregators implement $S_{1}$ or $S_{2}$ at the operation time.

8) The DSO settles the swap.

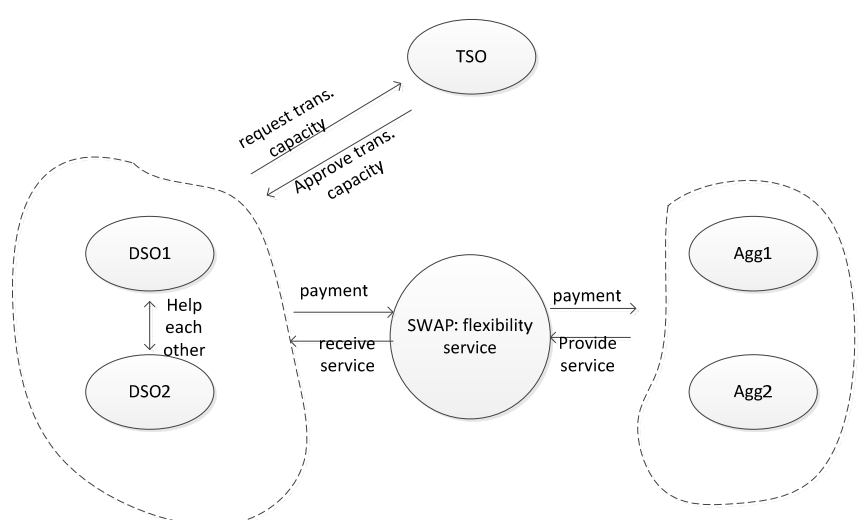

Fig. 2. Concept of the swap market

\section{ALGORITHM FOR FORMING SWAP}

In this section, an algorithm for forming the swap is proposed. As shown in section III.A, among the other parameters of the swap, the congestion time $t_{1}$ is identified by the DSO at first; otherwise, there is no need of swap. The amount of the exchange power $p$ is standardized to a fixed number, e.g. 100 $\mathrm{kW}$ or $50 \mathrm{~kW}$, to ease the programming. The size of the standardized exchange power is chosen such that the aggregators are easy to manage their flexible demands to provide the DR. The case with non-standardized $p$ can be derived from the standardized case. The time of the swap, e.g. $t_{1}$ and $t_{2}$, is indexed with a fixed duration, e.g. 30 minutes. The set of the time periods that the algorithm will search is denoted as $\mathcal{T}=\{1,2,3, \ldots n\}$ and $t_{1}=1$. The goal of the algorithm is to identify the suitable load points and $t_{2}$. The steps of the algorithm are presented as follows.

1) Step 1: Generate multiple solutions that can alleviate the congestion

In order to maximize the chance of the participation from the aggregators, it is desirable to have as many suitable load points as possible in $S_{1}$. Employ the following OPF, a MILP problem, to search for a feasible solution that can alleviate the identified congestion. 
OPF1:

$$
\min \sum_{i \in \mathcal{I}, j \in \mathcal{N}, t=1} x_{i j t}
$$

Subject to,

$$
\begin{gathered}
\sum_{i \in \mathcal{I}, j \in \mathcal{N}}-p D_{j} x_{i j t} \leq f_{t}, t=1 \\
\sum_{i \in \mathcal{I}, j \in \mathcal{N}} p D_{j} x_{i j t} \leq f_{t}, t>1, t \in \mathcal{T} \\
V_{0}\left(1-\frac{1}{V_{0}^{2}} \sum_{j^{\prime} \in \mathcal{N}}\left(\left(p_{j^{\prime} t}^{b}+p \sum_{i \in \mathcal{I}} x_{i j^{\prime} t}\right) R_{j j^{\prime}}+q_{j^{\prime} t}^{b} X_{j j^{\prime}}\right)\right) \geq V, \\
\forall j \in \mathcal{N}, t>1, t \in \mathcal{T} \\
\sum_{j \in \mathcal{N}} x_{i j t} \leq 1, i \in \mathcal{I}, t=1 \\
\sum_{t>1, t \in \mathcal{T}} x_{i j t}=x_{i j 1}, i \in \mathcal{I}, j \in \mathcal{N} \\
x \in\{0,1\}
\end{gathered}
$$

In OPF1, $\mathcal{I}$ is the set of swaps (one swap has a standardized exchange power $p$ ), $\mathcal{N}$ is the set of nodes, $x$ is the indicator of whether the load point is selected, $D$ is the power transfer distribution factor (PTDF), $f$ is the remaining capacity of the line loadings (negative means the corresponding line is overloaded).

Parameters $R_{j j}$ and $X_{j j}$ are the real and imagine parts of the elements in matrix $Z$. Matrix $Z$ is the inverse matrix of the partial nodal admittance matrix $Y_{L L}$, which is a submatrix of the admittance matrix of the distribution network,

$$
Y=\left[\begin{array}{ll}
Y_{00} & Y_{0 L} \\
Y_{L 0} & Y_{L L}
\end{array}\right] .
$$

The objective function (1) is to minimize the total number of swaps, which is also to minimize the cost of the swaps, since the cost of a standardized swap is fixed. Constraints (2)-(3) are power flow constraints, noticing that the swap is to reduce power at $t_{1}$ and to increase power at $t_{2}$. Constraint (4) calculates voltage according to an approximation method proposed in [26] and tested in [8]. Constraint (5) means that only one load point is selected in one swap. Constraint (6) means that the power increase is the same as the power decrease at the same load point, which is the idea of the swap.

After solving OPF1, a solution is found. The solution tells how many swaps are needed. For instance, the DSO is willing to use a maximum of 10 swaps to solve the congestion by defining $\mathcal{I}=\{1,2, \ldots, 10\}$. If the solution has an objective value 2 , it means that it only needs two swaps to solve the congestion. The solution also tells a candidate $S_{1}$ with e.g. two swaps: $S_{1}^{(1)}=\left\{L P_{j_{1}}, 1,-p, t_{2}^{(1)},+p\right\}$ and $S_{1}^{(2)}=\left\{L P_{j_{2}}, 1,-p, t_{2}^{(2)},+p\right\}$. Due to the symmetry of OPF1, the order of $S_{1}^{(1)}$ and $S_{1}^{(2)}$ does not matter.

Then exclude the above solution by adding new constraints to OPF1, e.g. if add new constraint $x_{i^{\prime} j^{\prime} t^{\prime}}+x_{i^{\prime \prime} j^{\prime \prime} t^{\prime \prime}} \leq 1$, then
$X_{i^{\prime} j^{\prime} t^{\prime}}$ and $x_{i " j "{ }^{\prime \prime}}$ cannot be one at the same time. Solve OPF1 again and find a new solution if any. Repeat the procedure till there is no new solution or the objective value starts to increase or the total number of solutions reaches a predefined number, e.g. 100.

\section{2) Step 2: Select $t_{2}$}

Theoretically, one side of the swap, $S_{1}$, can be formed as a group of all optimal solutions found in Step 1. For instance, if two swaps are needed to solve the congestion, the form can be,

$$
\begin{aligned}
S_{1}^{(1)}= & \left\{L P_{1}, t_{1},-p, t_{2}^{\prime}, p ;\right. \\
& L P_{3}, t_{1},-p, t_{2}^{\prime \prime}, p ; \\
& \left.L P_{11}, t_{1},-p, t_{2}^{\prime \prime \prime}, p ; \ldots\right\} \\
S_{1}^{(2)}= & \left\{L P_{2}, t_{1},-p, t_{2}^{*}, p ;\right. \\
& L P_{2}, t_{1},-p, t_{2}^{* *}, p ; \\
& \left.L P_{7}, t_{1},-p, t_{2}^{* * * *}, p ; \ldots\right\}
\end{aligned} .
$$

However, in order to reduce the complexity of forming $S_{2}$, the optimal solutions are further refined. To do so, $t_{2}$ is identified as an unordered $n$-tuple ( $n$ is the optimal value of OPF1) which has the maximum appearance in all optimal solutions found in Step 1. Form $S_{1}$ as the group of the optimal solutions having the selected $t_{2}$ and drop the rest of the optimal solutions. For instance, if $t_{2}^{\prime}=t_{2}^{\prime \prime} \neq t_{2}^{\prime \prime \prime}$ and $t_{2}^{*}=t_{2}^{* *} \neq t_{2}^{* * *}$, then $\left(t_{2}^{\prime}, t_{2}^{*}\right)$ is such 2-tuple having maximum appearance. In this way, the form of the $S_{1}$ can be merged and simplified as,

$$
\begin{aligned}
& S_{1}^{(1)}=\left\{L P_{1,3}, t_{1},-p, t_{2}^{\prime}, p\right\}, \\
& S_{1}^{(2)}=\left\{L P_{2}, t_{1},-p, t_{2}^{*}, p ;\right\} .
\end{aligned}
$$

3) Step 3: Form $S_{2}$

Employ the following OPF with a dummy objective function to find a candidate $S_{2}$.

OPF2: $\min 0$

Subject to,

$$
\begin{gathered}
\sum_{i \in \mathcal{I}^{*}, j \in \mathcal{N}} p D_{j} y_{i j t} \leq \tilde{f}_{t}, t=1 \\
\sum_{i \in \mathcal{I}^{*}, j \in \mathcal{N}}-p D_{j} y_{i j t} \leq \tilde{f}_{t}, t>1, t \in \mathcal{T}^{*} \\
V_{0}\left(1-\frac{1}{V_{0}^{2}} \sum_{j^{\prime} \in \mathcal{N}}\left(\left(p_{j^{\prime} t}^{b^{*}}+p \sum_{i \in \mathcal{I}^{*}} y_{i j^{\prime} t}\right) R_{j j^{\prime}}+q_{j^{\prime} t}^{b} X_{j j^{\prime}}\right)\right) \geq V, \\
\forall j \in \mathcal{N}, t=1 \\
\sum_{j \in \mathcal{N}} y_{i j t}=1, i \in \mathcal{I}^{*}, t=1 \\
y_{i j t_{2}^{(i)}}=y_{i j 1}, i \in \mathcal{I}^{*}, j \in \mathcal{N} \\
y \in\{0,1\}
\end{gathered}
$$

In OPF2, $\mathcal{I}^{*} \subseteq \mathcal{I}$ is the set of active swaps according to $S_{1}$, $\mathcal{T}^{*} \subseteq \mathcal{T}$ is the set of active time periods according to $t_{2}$ in $S_{1}$, 
$y$ indicates whether the load point is selected in the swap, $\tilde{f}$ is the minimum remaining capacity of the line loadings after activating $S_{1}$ (as shown in Steps 1 and 2, $S_{1}$ has a group of selected candidates).

Constraints (8)-(9) are power flow constraints. Constraint (10) is from voltage limits. Constraint (11) means that the number of selected load point is precisely one in one active swap. Constraint (12) is to match $t_{2}$ in $S_{2}$ and $S_{1}$ such that the system balance is maintained.

Similar to Step 1, after finding one candidate $S_{2}$, exclude the solution by adding new constraints to OPF2 and find new candidates. Repeat till there is no feasible solution or the number of candidates reaches a predefined number. In this way, $\mathrm{S}_{2}$ is formed as the group of all possible candidates.

If there are not enough candidates within one DSO to form $S_{2}$ or it fails to attract the aggregators to take the formed $S_{2}$, the DSO should send a request to other DSOs. The other DSO can also employ OPF2 to find candidates and then form $S_{2}$ within its distribution network.

\section{CASE STUDY}

Case studies have been carried out using the distribution network shown in Fig. 1. Parameters of feeder 1, where the congestion occurs, are listed in Tables I and II. The EV availability, implying EV is parked at home and connected, is shown in Fig. 3, which is from the driving pattern study in [27]. Assume each household has one EV and one HP. It is a typical winter day and almost all HPs are running.

TABLE I

LOAD POINT DATA

\begin{tabular}{llll}
\hline $\begin{array}{c}\text { load } \\
\text { points }\end{array}$ & $\begin{array}{c}\text { customer } \\
\text { type }\end{array}$ & $\begin{array}{c}\text { peak conv. } \\
\text { load per } \\
\text { point (kW) }\end{array}$ & $\begin{array}{c}\text { number of } \\
\text { customers } \\
\text { per point }\end{array}$ \\
\hline LP1-LP4 & residential & 886.9 & 200 \\
\hline LP5 & residential & 813.7 & 200 \\
\hline LP6,LP7 & commercial & 671.4 & 10 \\
\hline
\end{tabular}

TABLE II

Key PARAmETERS OF THE CASE STUdy

\begin{tabular}{ll}
\hline \multicolumn{1}{c}{ parameter } & \multicolumn{1}{c}{ value } \\
\hline EV battery size & $25 \mathrm{kWh}$ \\
\hline Peak charging power & $11 \mathrm{~kW}(3$ phase $)$ \\
\hline Energy consumption per km & $150 \mathrm{Wh} / \mathrm{km}$ \\
\hline Minimum SOC & $20 \%$ \\
\hline Maximum SOC & $85 \%$ \\
\hline price of swap & $2 \mathrm{DKK} / \mathrm{kWh}$ \\
\hline L2 limitation & $1400 \mathrm{~kW}$ \\
\hline L3 limitation & $7000 \mathrm{~kW}$ \\
\hline L4 limitation & $1700 \mathrm{~kW}$ \\
\hline
\end{tabular}

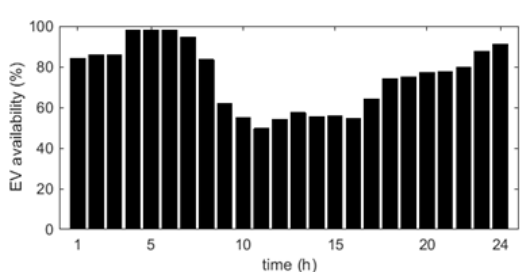

Fig. 3. EV availability

It is assumed that the line loadings resulting from the day-ahead planning, including conventional load, EV load and HP load, are respecting the line loading limits as shown in Fig. 4. The peak conventional consumption of residential customers occurs at 18:00 when people come home and start cooking or using other appliances (shown in Fig. 4).

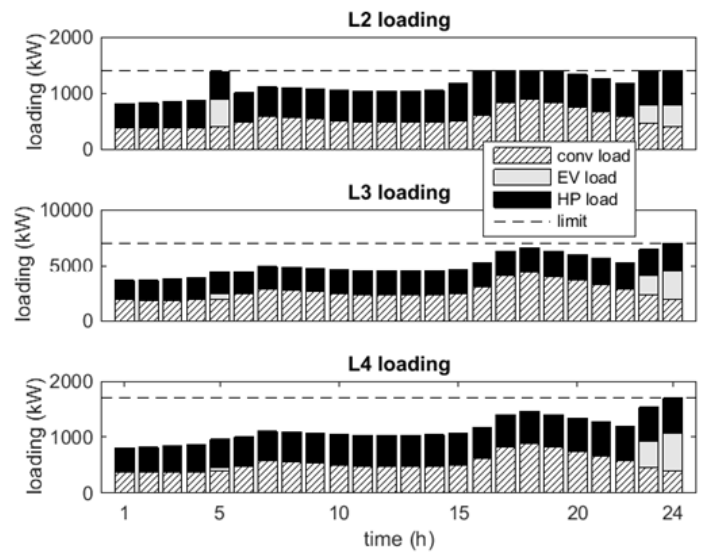

Fig. 4. Line loading resulting from the day-ahead planning

Two cases are presented in the following two subsections. One case illustrates the fundamental idea of the swap method using a straightforward example, where the stakeholders of the swap are all in one distribution network. The second case has a rather complicated situation, where a remote DSO and remote aggregators are involved. In this case, the algorithm of forming the swaps is employed.

\section{A. Case one}

As approaching the real operation time, e.g. 18:00, the DSO finds that there will be a overloading of L2 by the amount of $100 \mathrm{~kW}$ and the overloading will last for 30 minutes due to a wrong forecast, e.g. the conventional consumption will be more than the day-ahead forecast, or a loss of generation from a wind power generator. The forecast line loadings are shown in Fig. 5, where each period has 30 minutes and there are 12 periods in total. The DSO has to solve the congestion and it considers buying flexibility services from the aggregators since they are managing a large number of EVs and HPs in this distribution network.

As it is a simple case, the DSO finds out by observation that it needs a reduction of consumption at $18: 00$ by $100 \mathrm{~kW}$ on L2 and an increase of consumption at 22:00 by the same amount, because the flexibility services need to restore their batteries or household temperatures and there are enough free capacity at 22:00 on L2 as shown in Fig. 5. The DSO observes that there is 
enough free capacity of other lines of feeder 1 and all lines of other feeders to implement $S_{2}$. Hence a swap can be formed within this distribution network, which is (the standardized exchange power is assumed to be $100 \mathrm{~kW}$ in the case study, see the beginning of Section IV):

$$
S_{1}:\left\{L P_{1}\right\},(18: 00,30),-100,(22: 00,30),+100 \text {, }
$$
and,

$$
S_{2}:\left\{L P_{2-7}, L P_{11-38}\right\},(18: 00,30),+100,(22: 00,30),-100
$$

L2 loading
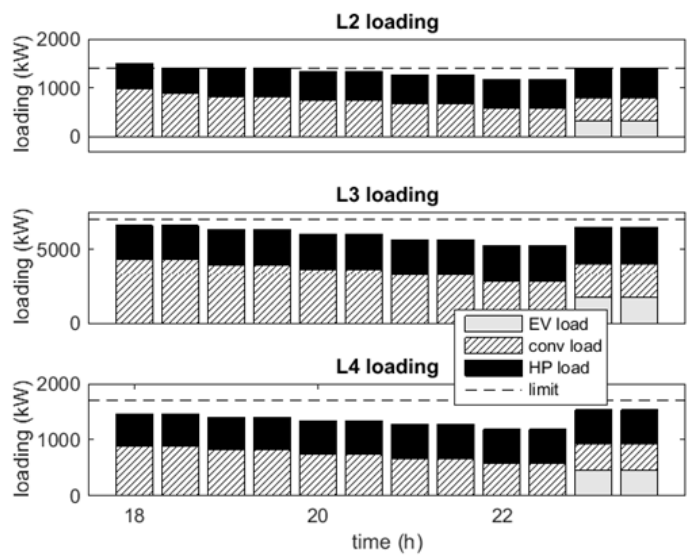

Fig. 5 Forecasted line loadings when close to the operating time

Because of the high HP availability indicated by high HP consumption as shown in Fig. 5, there is an aggregator taking both sides of the swap. After the confirmation of the swap from the DSO, the aggregator can activate the swap at the operation time. The aggregator will reduce HP consumption at LP1, which will result a reduction of consumption of L2. At the same time, the aggregator will increase HP consumption at LP2 in order to activate $S_{2}$ and maintain the system balance. Similarly, at 22:00, the aggregator will increase the HP consumption at LP1 to recover the household temperature. At the same time, the aggregator will reduce HP consumption at LP2 to maintain the system balance. The line loadings of L2, L3 and L4 after activating the swap are shown in Fig. 6, from which it can be seen that the congestion is solved.
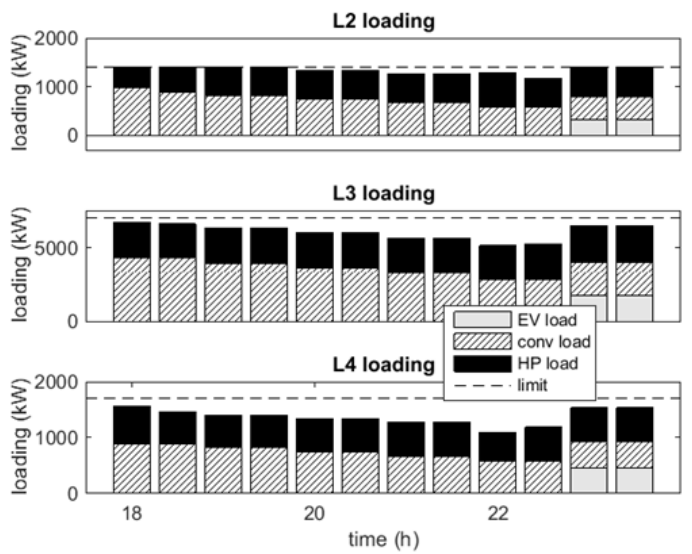

Fig. 6 Line loadings after activating swaps by HP DR
It is also possible to solve the congestion by the EV DR. For instance, assume that an EV aggregator is willing to accept the swaps. Because there is no EV consumption at 18:00 or 22:00, it is necessary to feed power back to the grid by discharging the battery (V2G), which has the same effect as reducing consumption of the HP. The line loadings of L2, L3 and L4 after activating the EV DR are shown in Fig. 7, from which it can be seen that the congestion is solved. The EV loading of L2 at 18:00 and the EV loadings of L3 and L4 at 22:00 are negative, which means that they discharge their batteries.
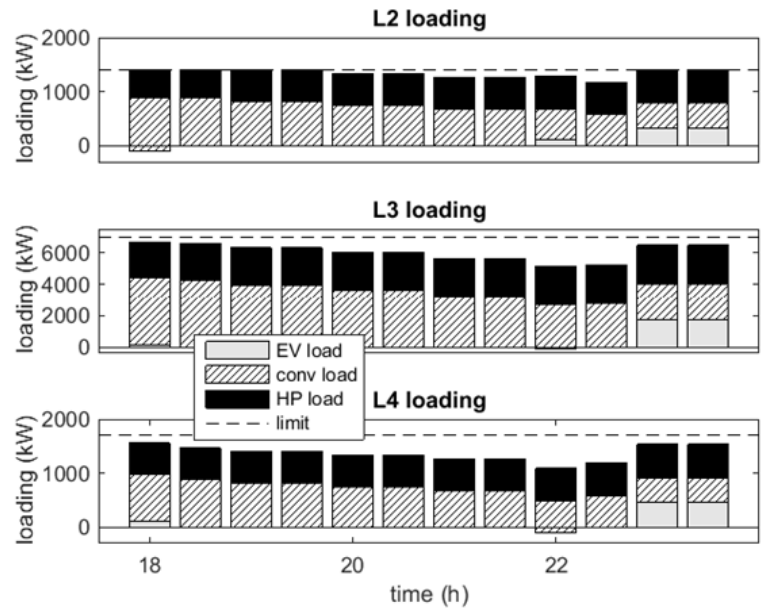

Fig. 7. Line loadings after activating swaps by EV DR

With either the HP DR or the EV DR, the swap is settled by a payment made by the DSO with the amount $2 p \delta s=2 \cdot 100 \cdot 0.5 \cdot 2=200 \mathrm{DKK}$. The aggregator makes a profit of 200 DKK in this case by providing flexibility services to solve the congestions in the distribution network.

If the FCH or DRX method is employed instead of the swap method, the DSO will just buy a DR to reduce the consumption at the congestion node. The DSO will not buy a DR to increase the consumption and maintain the system balance since the DSO is not responsible for it. In addition, the DR providers will need to consume more power in a future time, which will cause many economic and technical uncertainties to both the system operators and the DR providers.

\section{B. Case Two}

In Case Two, the algorithm for forming swaps is tested and the swap settlement involving remote DSO is considered. The DSO makes a forecast before 18:00 and the forecasted line loadings are shown in Fig. 8. Both L3 and L4 are overloaded: L3 is overload by $150 \mathrm{~kW}$ and L4 is overloaded by $60 \mathrm{~kW}$. Because one swap has a standard $100 \mathrm{~kW}$ power exchange capacity (assumption), multiple swaps are employed. The algorithm of forming the swaps consists of three steps.

Step 1: OPF1 is employed to search for the first candidate load points and $t_{2}$. The minimum number of swaps is two, denoted as $S_{1}^{(1)}$ and $S_{1}^{(2)}$, after solving OPF1. Then exclude the candidate and search for all other candidates. In the end, in total 100 candidates are found after 100 iterations. The algorithm is implemented in General Algebraic Modeling System (GAMS) 
[28], and the CPU (a laptop intel i5 4-core CPU) time is recorded and shown in Fig. 9. The total time is about 35 seconds.
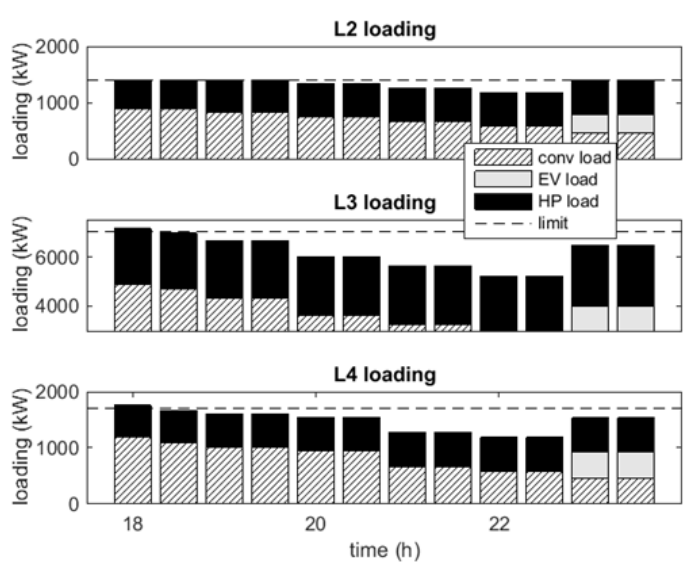

Fig. 8 The forecast line loadings when close to 18:00

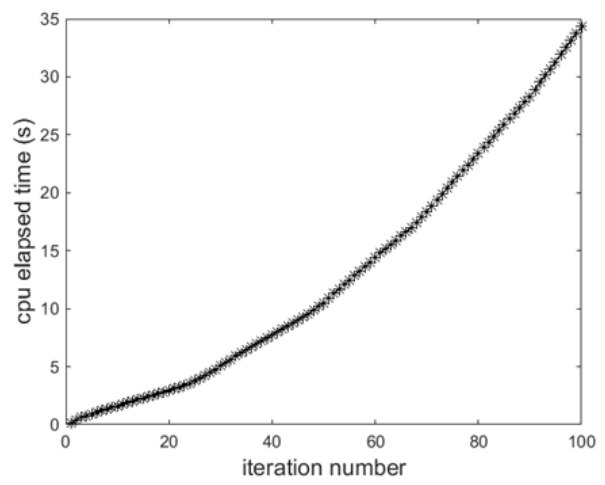

Fig. 9. CPU elapsed time for forming one side of the swap

Step 2: Observing all candidates, it is found that 20:00 and 20:30 are good time periods for consumption increase and load points 2 5 are suitable for activating $S_{1}$. And LP2 must be in one of the two swaps. To simplify the business between the DSO and the aggregators, the DSO decides to fix LP2 in $S_{1}^{(1)}$ with $t_{2}=20: 00$. All the actions in this step can be done by designing a searching program, but it is not included in this paper for brevity.

Hence, one side of the two swaps are formed as,

$$
S_{1}^{(1)}:\left\{L P_{2}\right\},(18: 00,30),-100,(20: 00,30),+100
$$

and,

$$
S_{1}^{(2)}:\left\{L P_{2-5}\right\},(18: 00,30),-100,(20: 30,30),+100 \text {. }
$$

Step 3: In order to form $S_{2}$, the DSO solves OPF2. It can be seen from Fig. 8 that there is no free capacity on L2 at 18:00. Assume that there is no free capacity on other feeder either. Therefore, OPF2 returns no feasible candidate. The CPU time of solving OPF2 is at the same level of solving OPF1 due to similar complexity; therefore, the total time for forming the swap is about 1 minute to several minutes and suitable for real-time congestion management.

The DSO will then broadcast a request to neighboring and/or remote DSOs. The request can be,

$$
\begin{aligned}
& S_{2}^{(1)}:\left\{L P_{\times}\right\},(18: 00,30),+100,(20: 00,30),-100, \\
& \text { and, } \\
& S_{2}^{(2)}:\left\{L P_{\times}\right\},(18: 00,30),+100,(20: 30,30),-100 .
\end{aligned}
$$

Assume that there are a number of DSOs that have enough free capacity to implement $S_{2}$. They send the information to the DSO with congestion and the TSO. The TSO validates the technical limit of the transmission lines and rejects some candidate DSOs. The remaining DSOs will broadcast $S_{2}$ to attract potential interested aggregators in their own networks.

Finally, assume that one aggregator takes $S_{1}$ and another aggregator takes $S_{2}$. The line loadings of the local distribution network after activating $S_{1}$ are shown in Fig. 10, where there is no congestion. The line loadings of the remote distribution network after activating $S_{2}$ are also respecting the limits of the corresponding network.



Fig. 10. Expected line loadings after activating $S_{1}$

The settlement of the two swaps depends on the market setup: single price market, zonal price market or nodal price market. For the single price market, the settlement will be the same as the situation in case one, i.e. the DSO should pay the two aggregators according to the swap price and the number of the swaps taken by the aggregators. For the nodal price market, the DSO should not only pay the cost of the swap as for the single price market, but also need to cover the possible losses due to different energy prices at $t_{1}$ and $t_{2}$. For instance, assume that the first aggregator has a profit/loss $a_{1}$ due to activating $S_{1}$ and the second aggregator has a profit/loss $a_{2}$. Then the DSO needs to pay $\max \left(0,-a_{1}-a_{2}\right)$ to the two aggregators. This means if the two aggregators have a net loss, it will be covered by the DSO. But if the two aggregators have a net profit, one of the aggregators can keep the net profit.

For the zonal price market, if the neighboring/remote distribution network is within the same price zone with the local distribution network, the settlement will be the same as in the single price market. Otherwise, the settlement will be the same as in the nodal price market. 


\section{CONCLUSIONS}

This paper proposes a real-time congestion management method by swap of EV charging and HP consumption. By reducing consumption at the congestion points while increasing the same amount of consumption at other points, the total power balance is maintained and the congestion can be solved. There is no rebound issue because the reverse consumption in a predefined future time is allowed by the swap to recover the batteries of EVs or household temperatures. The settlement of the swap is the payment made by the DSO based on the price of the swap, and the amount and duration of power being activated. The cost or profit resulting from the regulation power market is neutralized by a special agreement made between the DSO and aggregators. In the future work, the energy losses of the EVs and HPs due to the swap activating, and the transmission line losses due to the swap activating with a remote DSO will be studied.

\section{REFERENCES}

[1] P. M. Sotkiewicz and J. M. Vignolo,"Nodal pricing for distribution networks: Efficient pricing for efficiency enhancing DG,” IEEE Trans. Power Syst., vol.21, no.2, pp. 1013-1014, May 2006.

[2] R. K. Singh and S. K. Goswami,“Optimum allocation of distributed generations based on nodal pricing for profit, loss reduction, and voltage improvement including voltage rise issue,” Int. J. Electr. Power Energy Syst., vol.32, no.6, pp. 637-644, Jul. 2010.

[3] F. Meng and B. H. Chowdhury,"Distribution LMP-based economic operation for future smart grid,” in Proc. 2011 IEEE Power and Energy Conference at Illinois, pp. 1-5.

[4] N. O’Connell, Q. Wu, J. Østergaard, A. H. Nielsen, S. T. Cha, and Y. Ding,"Day-ahead tariffs for the alleviation of distribution grid congestion from electric vehicles,” Electr. Power Syst. Res., vol.92, pp. 106-114, 2012.

[5] R. Li, Q. Wu, and S. S. Oren,"Distribution locational marginal pricing for optimal electric vehicle charging management,” IEEE Trans. Power Syst., vol.29, no.1, pp. 203-211, Jan. 2014.

[6] S. Huang, Q. Wu, S. S. Oren, R. Li, and Z. Liu,“Distribution locational marginal pricing through quadratic programming for congestion management in distribution networks,” IEEE Trans. Power Syst., vol.30, no.4, pp. 2170-2178, Jul. 2015.

[7] S. Huang, Q. Wu, L. Cheng, and Z. Liu,“Optimal reconfiguration-based dynamic tariff for congestion management and line loss reduction in distribution networks,” IEEE Trans. Smart Grid, vol.7, no.3, pp. 12951303, May 2016.

[8] S. Huang and Q. Wu, "Dynamic subsidy method for congestion management in distribution networks,” IEEE Trans. Smart Grid, in press.

[9] P. Bach Andersen, J. Hu, and K. Heussen,"Coordination strategies for distribution grid congestion management in a multi-actor, multi-objective setting," in Proc. 3rd IEEE PES Innovative Smart Grid Technologies Europe (ISGT Europe), pp. 1-8.

[10] J. Hu, S. You, M. Lind, and J. Ostergaard,"Coordinated charging of electric vehicles for congestion prevention in the distribution grid," IEEE Trans. Smart Grid, vol.5, no.2, pp. 703-711, Mar. 2014.

[11] B. Biegel, P. Andersen, J. Stoustrup, and J. Bendtsen, “Congestion management in a smart grid via shadow prices," in Proc. 2012 8th IFAC Symposium on Power Plant and Power System Control, pp. 518-523.

[12] Z. Tan, P. Yang, and A. Nehorai,“An optimal and distributed demand response strategy with electric vehicles in the smart grid," IEEE Trans. Smart Grid, vol.5, no.2, pp. 861-869, Mar. 2014.

[13] L. Gan, U. Topcu, and S. H. Low, “Optimal decentralized protocol for electric vehicle charging,” IEEE Trans. Power Syst., vol.28, no.2, pp. 940-951, May 2013.

[14] H. Zhong, L. Xie, and Q. Xia,“Coupon incentive-based demand response: theory and case study,” IEEE Trans. Power Syst., vol.28, no.2, pp. 12661276, May 2013.

[15] M. R. Sarker, M. A. Ortega-Vazquez, and D. S. Kirschen,"Optimal coordination and scheduling of demand response via monetary incentives,” IEEE Trans. Smart Grid, vol.6, no.3, pp. 1341-1352, May 2015.

[16] C. Zhang, Y. Ding, N. Nordentoft, P. Pinson, and J. Østergaard,"FLECH-A danish market solution for DSO congestion management through DER flexibility services," J. Mod. Power Syst. Clean Energy, vol.2, no.2, pp. 126-133, 2014.

[17] K. Heussen, D. E. M. Bondy, J. Hu, O. Gehrke, and L. H. Hansen, “A clearinghouse concept for distribution-level flexibility services," in Proc. IEEE PES ISGT Europe 2013, pp. 1-5.

[18] Chunyu Zhang, Yi Ding, J. Ostergaard, H. W. Bindner, N. C. Nordentoft, L. H. Hansen, P. Brath, and P. D. Cajar,“A flex-market design for flexibility services through DERs,” in Proc. IEEE PES ISGT Europe 2013, pp. 1-5.

[19] D. T. Nguyen, M. Negnevitsky, and M. de Groot,"Pool-based demand response exchange-concept and modeling,” IEEE Trans. Power Syst. vol.26, no.3, pp. 1677-1685, Aug. 2011.

[20] D. T. Nguyen, M. Negnevitsky, and M. de Groot,"Walrasian marke clearing for demand response exchange,” IEEE Trans. Power Syst., vol.27, no.1, pp. 535-544, Feb. 2012.

[21] H. Wu, M. Shahidehpour, A. Alabdulwahab, and A. Abusorrah, "Demand response exchange in the stochastic day-Ahead scheduling with variable renewable generation," IEEE Trans. Sustain. Energy, vol.6, no.2, pp. 516-525, Apr. 2015.

[22] Y. Ding, S. Pineda, P. Nyeng, J. Ostergaard, and E. M. Larsen,"Real-time market concept architecture for EcoGrid EU-A prototype for European smart grids,” IEEE Trans. Smart Grid, vol.4, no.4, pp. 2006-2016, Dec. 2013.

[23] A. J. Conejo, J. M. Morales, and L. Baringo, "Real-time demand response model,” IEEE Trans. Smart Grid, vol.1, no.3, pp. 236-242, Dec. 2010.

[24] N. Flatabo, G. Doorman, O. S. Grande, H. Randen, and I. Wangensteen,"Experience with the Nord Pool design and implementation,” IEEE Trans. Power Syst., vol.18, no.2, pp. 541-547, May 2003.

[25] R. N. Allan, R. Billinton, I. Sjarief, L. Goel, and K. S. So,“A reliability test system for educational purposes-basic distribution system data and results,” IEEE Trans. Power Syst., vol.6, no.2, pp. 813-820, May 1991.

[26] S. Bolognani and S. Zampieri, "On the existence and linear approximation of the power flow solution in power distribution networks," IEEE Trans. Power Syst., vol.31, no.1, pp. 163-172, Jan. 2016.

[27] Q. Wu, A. H. Nielsen, J. Østergaard, F. Marra, and C. Træholt,"Driving pattern analysis for electric vehicle (EV) grid integration study,” in Proc. 2010 IEEE PES Innovative Smart Grid Technologies Conference Europe (ISGT Europe), pp. 1-6.

[28] R. E. Rosenthal. (2014, Aug. ). GAMS - A user's guide. GAMS Development Corporation, Washington, DC, USA. [online]. Available: http://www.gams.com/dd/docs/bigdocs/GAMSUsersGuide.pdf.

\section{IX. BIOGRAPHIES}



Shaojun Huang (S'13) obtained the B. Eng. from Department of Electrical Engineering, Tsinghua University, Beijing, P. R. China, in 2001. He obtained the MSc from Department of Energy Technology, AAlborg University, Den-mark, in 2013.

Currently, he is pursuing his $\mathrm{PhD}$ degree in Centre for Electric Power and Energy, Department of Electrical Engineering, Technical University of Den-mark.

His research interests are congestion management distributed energy resources. for distribution networks with high penetration of

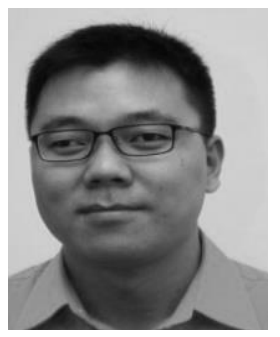

Qiuwei Wu (M’08-SM'15) obtained the B. Eng. and M. Eng. from Nanjing University of Science and Technology, Nanjing, P. R. China, in 2000 and 2003, respectively, both in Power System and Its Automation. He obtained the $\mathrm{PhD}$ degree from Nanyang Technological University, Singapore, in 2009, in Power System Engineering.

He was a senior R\&D engineer with Vestas Technology R\&D Singapore Pte Ltd from Mar. 2008 to Oct. 2009. He has been working at Department of Electrical Engineering, Technical University of 
Denmark (DTU) since Nov. 2009 (PostDoc Nov. 2009-Oct. 2010, Assistant Professor Nov. 2010-Aug. 2013, Associate Professor since Sept. 2013). He was a visiting scholar at Department of Industrial Engineering \& Operations Research (IEOR), University of California, Berkeley from Feb. 2012 to May

2012 funded by Ministry of Science, Technology and Innovation, Denmark. He is also a visiting professor named by Y. Xue, an Academician of Chinese Academy of Engineering, at Shandong University, China.

His research interests are modeling and control of wind power, electric vehicle, active distribution networks, electricity market and energy system integration. He is an Editor of IEEE Transactions on Smart Grid and IEEE Power Engineering Letters. He is also an Associate Editor of Inter-national Journal of Electrical Power and Energy Systems. 